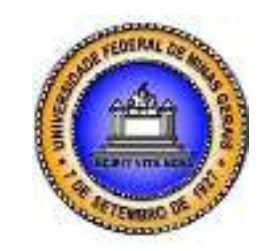

\title{
PARCERIAS PÚBLICO-PRIVADAS NA SAÚDE: O PROGRAMA PRO HOSP E SEUS INDICADORES DE RESULTADOS ${ }^{1}$
}

\section{PUBLIC-PRIVATE PARTNERSHIPS IN HEALTH: THE PROGRAM PRO HOSP AND ITS RESULTS INDICATORS}

\section{ASOCIACIONES PÚBLICO-PRIVADAS EN SALUD: EL PROGRAMA PRO HOSP Y SUS INDICADORES DE RESULTADOS}

\author{
ARLETE APARECIDA ABREU \\ IFMG Campus Formiga \\ arlete.abreu@ifmg.edu.br
}

RICARDO SOUZA SETTE

UFLA - Universidade Federal de Lavras

ricsouza@dae.ufla.br

\section{RESUMO}

As constantes transformações tanto sociais, quanto econômicas acabam por impulsionar adaptações tanto às empresas privadas quanto às instituições públicas. A intervenção estatal em diversos setores começa a se configurar com outras formas, modelos antes utilizados somente na gestão de entidades privadas. As parcerias público-privadas e o uso de indicadores de desempenho são um bom exemplo de mudanças deste tipo. Acompanhadas de ações específicas do governo, estas mudanças são direcionadas a setores em crise, como o setor de saúde. O Programa Pro Hosp e sua forma de avaliação e acompanhamento integram este arcabouço, podendo configurar-se como fortes indicativos de melhorias organizacionais. Por meio de índices que refletem a capacidade produtiva hospitalar, o programa Pro Hosp incentiva melhorias internas. Em contrapartida, os hospitais participantes recebem recursos financeiros repassados pelo governo de Minas Gerais, corroborando com mudanças tanto organizacionais quanto em sua estrutura física. Acredita-se na relevância do uso destas ferramentas, não só como forma de verificar mudanças propostas, mas como meio para detectar outros problemas a serem sanados.

PALAVRAS CHAVE: Pro Hosp. Saúde. Indicadores.

\section{ABSTRACT}

The constant economic and social changes drive changes to both private and public institutions. The state intervention in many sectors begins to take shape in other forms, previously used only in the private entities management. The public-private partnerships and the use of performance indicators are a example of such changes. Accompanied by specific government actions, these changes are directed to sectors in crisis, as the health sector. The Pro Hosp Program and their way of evaluation and monitor are part of this framework and can be configured as a stronger indicative of organizational improvements. Through indexes that reflect the productive capacity of the hospital, the Pro Hosp Program encourages internal improvements. In return, participating hospitals receive funds transferred by the government

1 Submetido em 06 de março de 2014. Aceito em 08 de Julho de 2014. O artigo foi avaliado segundo o processo de duplo anonimato e avaliado pelo editor. Editores responsáveis: Márcio Augusto Gonçalves e Lucas Maia dos Santos. Reprodução parcial ou total e trabalhos derivativos permitidos com a citação apropriada da fonte. 

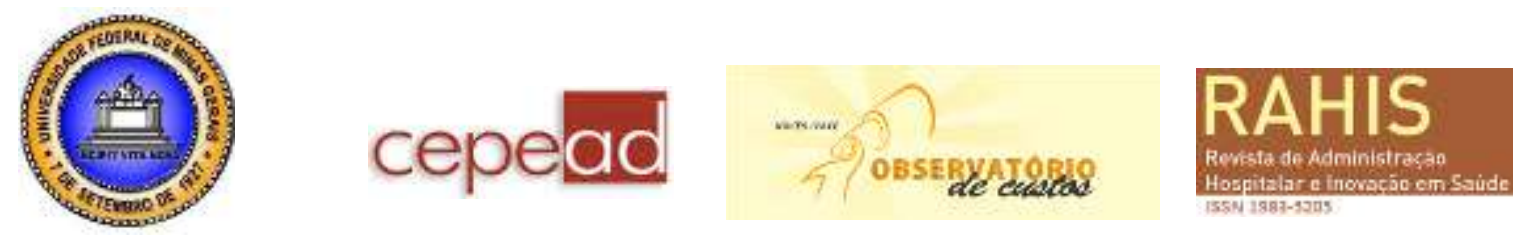

of Minas Gerais, confirming changes in both organizational and physical structure.It's believed in the importance of using these tools, not only as a way to verify the proposed changes, but as a means to detect other problems to be solved.

KEY WORDS: Pro Hosp. Health. Indicators

\section{RESUMEN}

Las constantes de ambas transformaciones sociales, económicas como en definitiva impulsar las adaptaciones a las dos empresas privadas e instituciones públicas. La intervención del gobierno en diversos sectores comienza a establecer con otras formas, los modelos utilizados hasta ahora sólo en la gestión de las entidades privadas. Las asociaciones público-privadas y el uso de indicadores de desempeño son un buen ejemplo de este tipo de cambio. Acompañado por acciones específicas del gobierno, estos cambios están dirigidos a sectores en crisis, como la industria de la salud. El Programa Hosp Pro y su forma de evaluación y seguimiento de este marco y se pueden configurar como fuertes predictores de mejoras organizativas. A través de los índices que reflejan la capacidad hospitalaria, el programa Pro Hosp alienta mejoras internas. A cambio, los hospitales participantes reciben fondos transferidos por el gobierno de Minas Gerais, lo que confirma tanto como los cambios

PALABRAS CLAVE: Pro Hosp. Salud. Indicadores

\section{INTRODUÇÃO}

As parcerias público-privadas (PPP's) representam uma nova forma de intervenção do Estado em setores vitais à população, sendo a saúde um destes. Trata-se de aplicar conceitos que compõem a chamada visão gerencialista à forma de gestão de bens públicos, ação adotada no Brasil a partir da década de 1990. O ideal da Nova Gestão Pública tem como eixo principal a utilização de ferramentas e modelos gerenciais antes praticados somente em instituições privadas, adequando-os às especificidades de setores governamentais, buscando melhorias em sua efetividade e abrangência.

Um dos objetivos primordiais da aplicação das PPP's ao contexto público é resgatar a capacidade de investimento em alguns setores sob responsabilidade do Estado, devido à dificuldade que o mesmo vem encontrando nesse quesito. Para Moreira Neto (2005) trata-se de um instrumento contratual gerado no quadro da globalização, do capitalismo ecumênico, do avanço tecnológico, combinados à insuficiência de recursos públicos, do repúdio ao aumento de tributos e à necessidade de recuperação das condições necessárias para a satisfação de demandas públicas, cada vez mais exigentes e complexas.

O programa Pro Hosp é um representante desse tipo de parceria, na medida em que, por meio da assinatura de contratos entre a SES (Secretaria Estadual de Saúde) e hospitais filantrópicos do estado de Minas Gerais, o Estado pactua metas e objetivos destinados à melhoria da prestação de serviços no setor de saúde. Diante disso, os hospitais são avaliados e o programa é monitorado por meio de indicadores de desempenho (outra prática advinda da administração de empresas privadas).

Estes indicadores, segmentados em cinco áreas, refletem algumas mudanças estabelecidas pelo programa, demonstrando redução/aumento de taxas que versam sobre o 

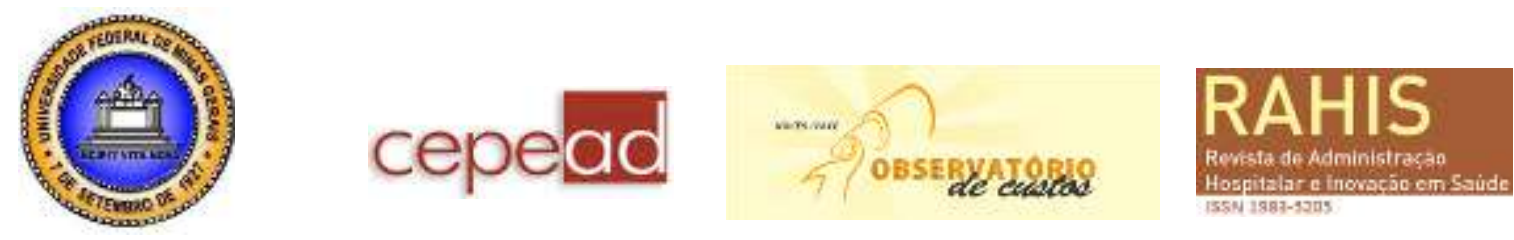

resultado da produção hospitalar (taxas de cesárea, mortalidade, ocupação, permanência e infecção).

Adotando-se o programa Pro Hosp como uma forma de parceria público-privada, o objetivo do presente artigo é demonstrar a evolução de alguns indicadores de desempenho utilizados pelo programa Pro Hosp por meio de uma tabela comparativa. Essa tabela de indicadores, além de demonstrar melhorias adotadas na prestação de serviços pelas instituições, também acaba por revelar o Pro Hosp como um meio de incentivo à produção de informações importantes para a gestão.

Partindo-se da perspectiva sobre a importância da inserção de conceitos empresariais à áreas públicas, especialmente o setor de saúde brasileiro, esta pesquisa pretende responder ao seguinte questionamento: Que melhorias podem ser observadas através da implantação de indicadores de desempenho adotados pelo Pro Hosp?

Portanto, o objetivo deste estudo é demonstrar, através da análise de indicadores de desempenho, que melhorias puderam ser constatadas em três hospitais filantrópicos, associados ao Pro Hosp, situados na região do Sul de Minas Gerais.

\section{Revisão Bibliográfica}

\section{As Parcerias Público-Privadas (PPPs): conceito e especificidades}

As parcerias público-privadas são uma forma de intervenção estatal no mercado, especificamente o mercado de saúde, com o objetivo não somente de regulá-lo, mas de contribuir para uma prestação de serviços mais igualitária e de qualidade. Este tipo de parceria se iniciou na Inglaterra, devido a desafios enfrentados por este país na busca de maneiras de fomentar investimentos, sem comprometer os escassos recursos públicos, sendo a iniciativa rapidamente difundida em outros países (PASIN; BORGES, 2003).

De forma geral, as parcerias público-privadas são o resultado de uma visão gerencialista adotada pelo governo, na qual o Estado é visualizado em termos de sua capacidade de produzir serviços economicamente sustentáveis. Trata-se de uma nova estratégia de recuperação da capacidade de investimento público (PECI; SOBRAL, 2006). Pode ser entendido como um tipo de relacionamento interorganizacional, construído no contexto da Nova Gestão Pública, com o objetivo de aumentar a eficiência, a qualidade e a competitividade dos serviços do setor público, enquanto resolve problemas macroeconômicos (LANE, 2000). "O governo precisa do setor privado para implementar seus projetos, pois estes possuem os recursos e investimentos necessários. Isso muda o relacionamento entre governo e mercado" (BULT-SPIERING; DEWULF, 2007, p. 1). Os arranjos formados pelas parcerias ressaltam e demonstram a incapacidade e as dificuldades enfrentadas pelo Estado para oferecer estrutura e serviços públicos de qualidade aos cidadãos.

Existe também uma conceituação de parceria público-privada presente no documento da Secretaria de Planejamento e Investimentos Estratégicos do Ministério de Planejamento, Orçamento e Gestão (MPOG), destinada especificamente à viabilização de projetos de infraestrutura. Trata-se de:

Acordos entre Governo e entidades do setor privado com a finalidade de prover, às comunidades, equipamentos de infraestrutura pública e 

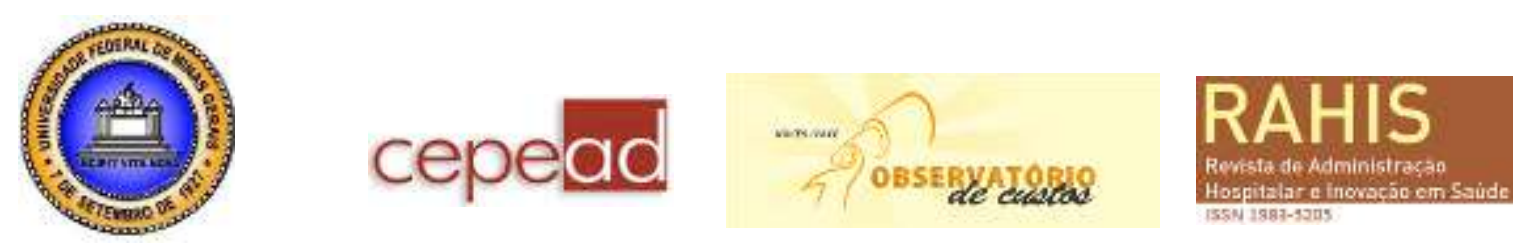

serviços correlatos. Tais parcerias são caracterizadas pela repartição compartilhada, entre os parceiros, dos investimentos, riscos, responsabilidades e ganhos. As condições para o estabelecimento de tais parcerias variam, mas geralmente envolvem o financiamento, projeto, construção, operação e manutenção dos equipamentos e serviços de infraestrutura (BRASIL, 2001 apud SOARES; CAMPOS NETO, 2002, p. 6).

Contudo, existe ainda certa confusão na definição do conceito deste tipo de parceria (AKINTOYE; BECK; HARDCASTLE, 2003; BULT-SPIERING; DEWULF, 2007), explicada por sua similaridade com as privatizações e pelo extenso número de tipos que elas podem representar. Segundo Klijn e Teisman (2003) as PPP's podem ser entendidas como um tipo de cooperação "mais ou menos" sustentável entre entidades públicas e privadas, em que produtos e serviços são desenvolvidos e riscos, custos e lucros são compartilhados. Já o conceito de privatização representa a "transformação de tarefas e responsabilidades para o setor privado, ficando ambos os custos e receitas em mãos privadas" (BULT-SPIERING; DEWULF, 2007, p. 3).

Especificamente no contexto brasileiro, as parcerias público-privadas representam formas estratégicas adotadas a partir da década de 1990, como parte integrante dos ideais da Reforma do Estado. Seu objetivo também foi o de alavancar setores que necessitam de aplicação de recursos.

As normas gerais para a contratação de PPP's foram editadas com a Lei 11.079/ 04, durante o governo do Presidente Luiz Inácio Lula da Silva. A lei que rege este tipo de parceria pode ser considerada como o resultado da insuficiência de um arcabouço composto pela Lei das Concessões ( $n^{\circ}$ 8.987, de 1995) e a reforma das Leis das Licitações e Contratos Administrativos ( $\mathrm{n}^{\circ} 8.666$, de 1993), além de outras leis de cunho regulatório e social que se mostraram insuficientes na atração de investimentos privados para áreas importantes. Estas leis visam viabilizar a gestão não exclusivamente estatal dos interesses públicos (SUNDFELD, 2005), compartilhando responsabilidades com o setor privado.

Pela importância e representatividade que vem adquirindo no cenário atual, as PPP's têm sido objeto de muitos questionamentos, tanto em diferentes áreas, quanto sob diferentes perspectivas: sob seu risco (ALONSO-CONDE; BROWN; ROJO-SUAREZ, 2007; FREDEBEUL-KREIN; KNOBEN, 2010; KE et al., 2010; TAKASHIMA; YAGI; TAKAMORI, 2010); aplicadas à construção civil (JONG et al., 2010; TANG; SHEN; CHENG, 2010); à indústria energética (MARTINS; MARQUES; CRUZ, 2011); à educação (QUAH; LIM; BROOK, 2010); ao transporte (EVENHUIS; VICKERMAN, 2010; MU; JONG; KOPPENJAN, 2011) à agricultura (KHAN; MUSHTAG, 2009; NARROD et al., 2009; POULTON; MACARTNEY, 2011).

$\mathrm{Na}$ área da saúde não poderia ser diferente. As PPP's também vêm se tornando fonte de mudanças na busca por uma gestão eficiente, adaptada a um cenário tão conturbado e envolto em crises. Estas parcerias objetivam não somente contribuir com a equalização da prestação de serviços em saúde, sua qualidade e efetividade, mas também influenciar o mercado e reduzir as disparidades geradas pelo gasto público. 

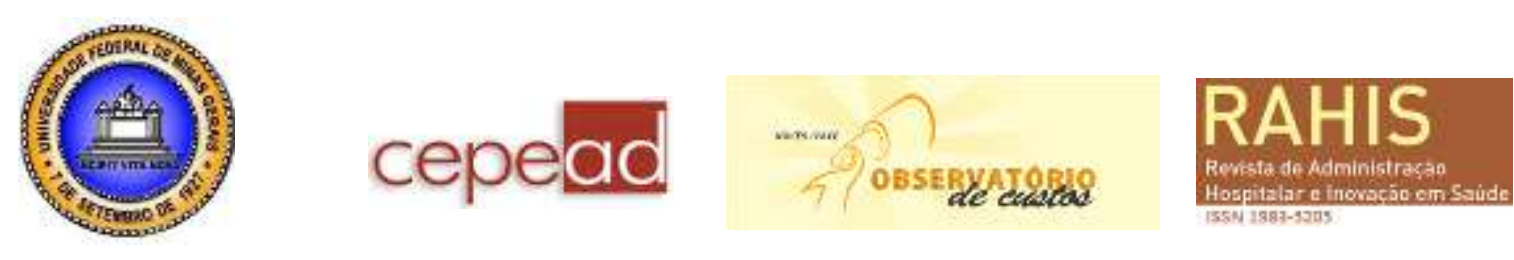

Galvão (1997) analisou onze empreendimentos em execução, com o objetivo de identificar as características de uma PPP. Um dos modelos de parceria observados foi a chamada "contratualização", cujas características foram assim definidas:

i) possibilidade de livre escolha do parceiro; ii) relação contratual negociada - objetivos, controles, metas, garantias, parâmetros de qualidade, financiamento, vigência; iii) Diversidade de tipos de contratos - convênio, protocolo de intenções, termo de compromisso, contrato inominado, contrato típico, etc.; iv) possibilidade da relação entre parceiros ser precedida por autorização legislativa e/ou ato do Poder Executivo, ou ainda ser estabelecida sem interferência desses poderes; v) natureza do parceiro - público ou privado com ou sem fins lucrativos (GALVÃO, 1997, p. 47).

Os contratos estabelecidos entre o setor público e o terceiro setor são os específicos para serviços sociais. Trata-se da parceria estabelecida com organizações da sociedade civil, para a prestação de serviços públicos (ALCOFORADO, 2005). O mesmo autor relata que a contratualização com as entidades dos terceiro setor pode ser instrumentalizada por meio de contratos de gestão ou termos de parceria, compostos por resultados, indicadores e metas que refletem os serviços que estão sendo contratados. Todas estas características são parte de uma gestão voltada para resultados, calcada na busca de melhorias mensuráveis para diversos setores que atendem a sociedade.

Nesse sentido, o governo de Minas destaca-se, tanto em relação aos princípios da NGP quanto em relação à adoção de parcerias público-privadas, especialmente na saúde. Por meio do Programa Pro Hosp, o governo de Minas Gerais incentiva os hospitais filantrópicos a prestarem um serviço de qualidade, mediante a assinatura de um termo de compromisso que especifica metas, sendo estas avaliadas por meio de indicadores de desempenho. O hospital que alcança as mudanças pretendidas recebe o incentivo financeiro.

Prezando pela eficiência e pelo menor custo dos serviços sociais oferecidos por instituições privadas, o governo combate a ideia de desperdício na administração pública. $\mathrm{O}$ Pro Hosp representa uma iniciativa do governo de Minas participante do conceito de PPP, influenciando o mercado de saúde, motivando a iniciativa privada e canalizando recursos em busca da eficiência.

\section{O Programa Pro Hosp: a aplicação da NGP e do conceito de PPP aos serviços de saúde}

A saúde brasileira tem nos hospitais um de seus principais componentes, respondendo por dois terços dos gastos do setor (LIMA et al., 2009). Esse dado revela a dimensão da importância das instituições hospitalares para a saúde no país. Segundo dados do Instituto Brasileiro de Geografia e Estatística - IBGE (2010) havia no Brasil 73.516 estabelecimentos de saúde que ofereciam atendimento ambulatorial/hospitalar, no ano de 2009.

De acordo com La Forgia e Couttolenc (2009), essa natureza "hospitalocêntrica" do país e a ênfase no tratamento de casos agudos têm deixado o Brasil despreparado para enfrentar a incidência crescente de doenças crônicas. Além disso, em relação ao contexto mineiro, Mendes (2007) afirma que os hospitais enfrentam sérios problemas, podendo estes 

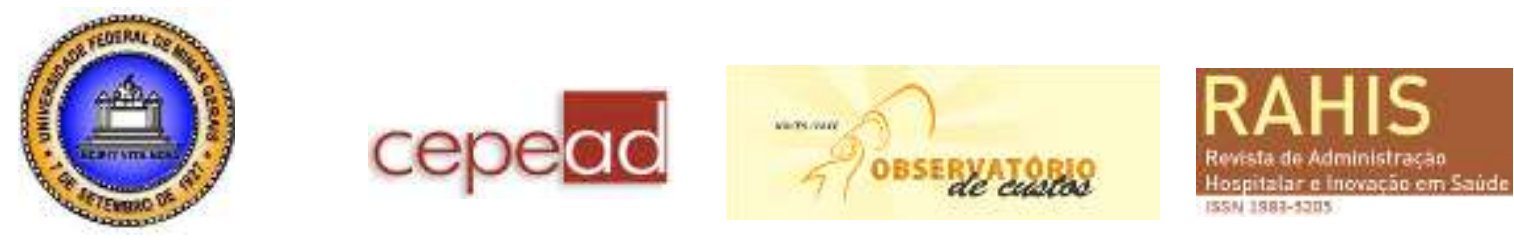

ser agrupados em três dimensões: a ineficiência, a baixa qualidade dos serviços ofertados e o subfinanciamento. Corroborando com a informação, em 2003, um estudo feito pelo Comitê Estratégico da SES/MG apontou uma rede hospitalar fragmentada e com unidades que estavam longe de constituir espaços efetivamente adequados à atenção hospitalar.

Desse modo, o governo de Minas agiu no intuito de implementar políticas que pudessem lidar com esses problemas e melhorar os serviços em saúde no estado. Por meio do chamado choque de gestão e da aplicação de princípios da NGP à saúde, Minas foi um estado pioneiro na formação de estratégias gerenciais que fossem voltadas ao setor. Uma dessas ações foi a implantação de um programa que fosse baseado em um sistema de parceria entre o governo do estado e os hospitais, visando à potencialização da qualidade no acesso aos serviços do SUS.

O Programa de Fortalecimento e Melhoria da Qualidade dos Hospitais do SUS/MG, ou Pro Hosp, foi concebido no intuito de consolidar a oferta em atenção hospitalar nos polos macro e microrregionais ${ }^{2}$ de Minas Gerais, com o desenvolvimento de um parque hospitalar para que o SUS opere com eficiência e qualidade, tendo como alvo os hospitais públicos e filantrópicos do estado (LIMA et al., 2009). De acordo com o PDR-SUS/MG -2003/2006 (Plano Diretor de Regionalização de Minas Gerais), estabelecido pelo choque de gestão, os polos microrregionais exercem força de atração para a demanda por serviços no nível de "atenção secundária", ofertando poucos procedimentos de "alta complexidade tecnológica". Já os polos macrorregionais exercem força de atração para alguns serviços, com oferta de procedimentos de "média complexidade", cuja demanda é rara e que exigem grande especialidade de diagnósticos. Os polos macrorregionais exercem grande força de atração para a maioria dos serviços de "alta complexidade" 3 .

A partir deste ponto, os hospitais não seriam mais considerados pontos isolados de atenção à saúde, mas seriam partes de redes de atenção integrando atenção primária, secundária e terciária (MENDES, 2007). Outro ponto importante salientado pelo autor foi a mudança dos convênios para os contratos de gestão, por meio de metas pactuadas em termos de compromisso, o que reafirma a característica de parceria dotada pelo programa.

O programa propõe uma alocação de recursos aos hospitais como um incentivo à mobilização de arranjos organizacionais e práticas de gestão interna, tendo como objetivos a eficiência, a equidade e a melhoria da qualidade do atendimento e dos serviços (LIMA et al., 2009). Muitos pequenos hospitais coexistiam com poucos hospitais com escala e densidade tecnológica suficiente para funcionar eficaz e eficientemente no enfrentamento de eventos agudos de saúde - razão de ser da atenção hospitalar (MENDES; SILVA, 2004).

\footnotetext{
${ }^{2}$ Os pólos macro e microrregionais de Minas foram estabelecidos em razão do choque de gestão e da necessidade de dividir o estado em regiões específicas para que essas pudessem ser afetadas pelas estratégias adotadas pela reforma. De acordo com o Plano Diretor de Regionalização 2003-2006, o território mineiro foi dividido segundo a complexidade da assistência envolvida e os fluxos intermunicipais. As microrregiões atendem a serviços de média complexidade hospitalar e ambulatorial (níveis 1 e 2), enquanto as macrorregiões atendem à média complexidade ambulatorial de nível 3 e à alta complexidade hospitalar e ambulatorial. No total, são 13 macrorregiões e 75 micro.

${ }^{3}$ A atenção primária em saúde refere-se aos serviços de "baixa complexidade". São serviços mais simples e que ficam à porta do sistema de saúde; são os cuidados preventivos. A atenção secundária são os serviços que se referem à "média complexidade". São aqueles tratamentos curativos e que se destinam a casos menos complexos. Já a atenção terciária refere-se aos serviços realizados em nível de "alta complexidade", como transplantes, hemodiálise, quimioterapias, etc.
} 

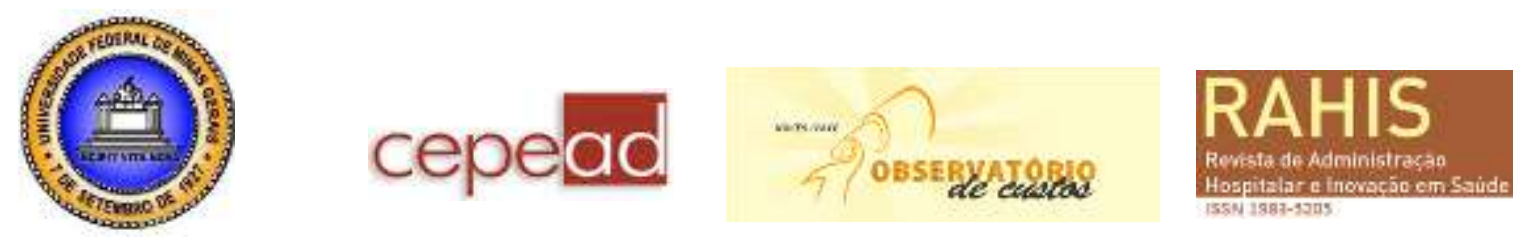

Por meio de metas e contratos de gestão, a política do Pro Hosp adentra o ambiente hospitalar, impulsionando melhorias na atenção à saúde e contribuindo com as mudanças tidas como necessárias pelo governo de Minas. O programa conta também com incentivo à capacitação de gestores e o monitoramento intensivo do desempenho do hospital, tendo em vista as metas pactuadas e o contrato de gestão entre a instituição e a Secretaria Estadual de Saúde. Assim, a cada exercício do programa, os hospitais elaboram um Plano de Ajustes e Metas (PAM), contendo seus compromissos e projetos para aplicação dos recursos.

O uso de contratos de gestão e o estabelecimento de acordos e metas são algumas maneiras pelas quais o programa Pro Hosp se utiliza para colocar em prática a política que defende. Molina (2002) afirma que o Pro Hosp pode ser considerado um programa de natureza interativa, em que vários atores participam do processo de execução e controle das metas, citando a Comissão Estadual de Avaliação do Pro Hosp, os comitês de avaliação macro e microrregional do Pro Hosp; as gerências regionais de saúde, os próprios hospitais que assinam o termo, as secretarias municipais de saúde, a Comissão Intergestores Bipartite, o conselho municipal de saúde e, por fim, o Conselho Estadual de Saúde. Desse modo, todos estes atores participam do programa, contribuindo para a sua execução, discutindo melhorias e repensando a saúde como um bem coletivo.

O monitoramento do programa se dá por meio de indicadores de desempenho hospitalar. Esses indicadores permitem o acompanhamento das metas e compromissos pactuados entre a instituição e a SES/MG, além de demonstrar ações que, embora não pactuadas, também reflitam a melhoria da qualidade na gestão e prestação de serviços hospitalares. Segmentados em cinco áreas temáticas, os indicadores acabam por demonstrar uma visão ampla do ambiente organizacional, sendo compostos por: indicadores referentes à gestão, indicadores referentes a processos, indicadores de resultados e indicadores referentes à relação com os usuários e com a comunidade.

Percebe-se, portanto, que o Pro Hosp representa o reflexo dos princípios da NGP, aplicados especificamente à saúde, ou seja, trata-se de pensar os serviços de saúde estrategicamente, buscando ações antes aplicadas somente à atividades de empresas privadas. Outros estudos nesta mesma vertente demonstram a aplicação destes ideais à prestação de serviços por parte do poder público, especificamente com relação às PPP's, à inserção da iniciativa privada na saúde e os ganhos em eficiência desta abordagem (MAYSTON, 1999).

Focando a aplicação destes princípios à gestão pública, Russel, Bennett e Mills (1999) analisaram a extensão e a profundidade da reforma ocasionada pela NGP em cinco países. Além disso, os autores revisaram as várias dimensões que impedem o desenvolvimento e a aplicação de políticas embasadas no ideal da mudança. Pesquisando os países de Gana, Zimbábue, Sri Lanka, Tailândia e Índia, os autores chegam à conclusão de que a aplicação da NGP não exige somente reformas complexas, mas uma liderança política forte, maiores mudanças institucionais e alterações na cultura organizacional.

A pesquisa de Russell, Bennett e Mills demonstra, portanto, a necessidade de mudanças mais concisas com os ideais da NGP. Acredita-se que esta constatação também se aplique ao contexto brasileiro, já que o país tem uma cultura que guarda resquícios de uma administração pública pouco racional e objetiva.

A descentralização, a gestão por resultados, as parcerias e os contratos, os acordos de metas e o aprimoramento profissional constituem o primeiro passo para alcançar os objetivos estratégicos do futuro. 

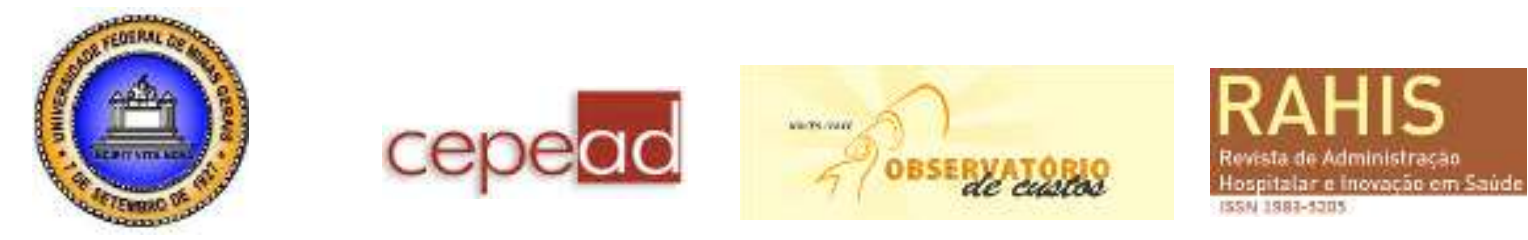

\section{Indicadores de resultados utilizados pelo Programa Pro Hosp: mensurando o desempenho hospitalar}

Medir o desempenho empresarial tornou-se prática mais comum a partir do momento em que os mercados tornaram-se globalizados e altamente competitivos. Trata-se da formação de um complexo sistema de informações que possam auxiliar nas decisões estratégicas das empresas. Os indicadores financeiros foram os primeiros a serem utilizados, especialmente em empresas como a Dupont e a General Motors, no início do século XX (NEELY; BOURNE, 2000).

Segundo Ghalayini e Noble (1996), a literatura que trata sobre as medidas de desempenho pode ser dividida em duas fases distintas: a primeira, por volta de 1880, na qual indicadores de desempenho financeiro, como medidas sobre o lucro e a produtividade, eram as mais utilizadas e a segunda fase, ocorrida por volta de 1980, quando empresas começaram a perder espaço no mercado, a concorrência começou a se acirrar, o que demonstrou que as medidas tradicionais de desempenho eram limitadas e precisavam ser repensadas. A partir deste momento, indicadores que envolviam outras informações relevantes para o processo decisório começaram a ser utilizadas.

A utilização de indicadores de desempenho empresarial passou a compor a agenda de ações destinadas à gestão de bens públicos a partir da década de 1990. Com a implantação da chamada Nova Administração Pública, gestores de vários níveis de governo puderam buscar, na administração de empresas privadas, elementos que agregassem valor aos serviços públicos.

A partir de então, indicadores de desempenho vêm sendo utilizados em diversos setores, como saúde, educação e saneamento, dentre tantos outros. Estes indicadores permitem um diagnóstico da realidade local e revelam grandes diversidades existentes, mesmo dentro do município e da região ao qual o indicador se refere (ANDERSEN, 2004). Podem, assim, ser considerados como uma ferramenta de avaliação das ações e políticas públicas destinadas a determinado setor da sociedade.

Utilizados como forma de avaliação, os indicadores de desempenho também compõem o arcabouço de ações destinadas às mudanças implementadas pelo choque de gestão em Minas Gerais. Por meio destes, o governo monitora as mudanças estabelecidas pelas instituições hospitalares que estabelecem um acordo de metas e resultados com o Pro Hosp. Segundo o próprio manual do programa,
A proposta do sistema de monitoramento pressupõe o acompanhamento tanto dos compromissos e metas estabelecidos entre o hospital e a SES-MG, decorrente do modelo contratual adotado, quanto de indicadores que, embora não pactuados, refletem a qualidade da assistência hospitalar (MINAS GERAIS, 2007, p. 22).

Os indicadores acabam por refletir as mudanças propostas por cada contrato de gestão e também por reafirmar o acordo e o comprometimento do hospital com o programa, uma vez que o não cumprimento de alguma metas implica em perda de recursos financeiros. Trata-se de uma forma de fazer cumprir a política implícita no programa, gerando os benefícios esperados e as mudanças estabelecidas. 


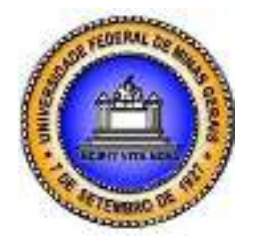

Para a avaliação e o monitoramento do Pro Hosp, os indicadores foram agrupados em cinco áreas temáticas: gestão, processos, relação com os usuários e com a comunidade, resultados e estrutura. A área denominada gestão abrange indicadores referentes à direção da instituição, à gestão dos recursos humanos, à gestão administrativo-financeira e à gestão da informação hospitalar.

A segunda área, denominada processos, abrange indicadores que demonstram mudanças na organização da assistência prestada e medidas com relação à produção, à produtividade e ao tempo de espera. A área que trata da relação com os usuários e com a comunidade trata de indicadores referentes à relação com o usuário e à interação da instituição com a comunidade. A área de resultados traz indicadores que retratam taxas importantíssimas e que refletem todo o processo dedicado à atenção dos pacientes: taxas de mortalidade, de infecção hospitalar e taxas sobre a movimentação de pacientes. Por fim, a quinta e última área abrange informações sobre a estrutura do hospital, sendo composta de indicadores que demonstrem informações gerais sobre a entidade e a adesão desta a programas oficiais do governo.

Estes indicadores demonstram um pouco o panorama organizacional em que cada instituição acordada com o programa se encontra. Refletem as mudanças alcançadas em alguns setores importantes da prestação de serviços como um todo, sendo, por este motivo, um bom indicativo de desempenho.

\section{METODOLOGIA}

Para a consecução deste estudo, foram pesquisadas três instituições associadas ao Programa Pro Hosp, ambas situadas em cidades ao sul do estado de Minas Gerais, configurando-se, portanto, como um estudo multicasos. Por questões de sigilo nas informações veiculadas, os hospitais serão identificados como Hospital X, Y e Z, possuindo 126, 78 e 136 leitos, respectivamente. Todos os três hospitais são enquadrados na natureza jurídica filantrópica, além de possuírem mais de 300 funcionários (307, 323 e 364, respectivamente). O critério de escolha das instituições baseou-se na facilidade de acesso aos dados e informações pertinentes ao estudo.

Para Vergara (2000), o estudo de caso é aquele circunscrito a uma ou poucas unidades, entendidas essas como uma pessoa, uma família, produto, empresa ou país. Pode ser também utilizado para estudar, além de uma pessoa, uma organização ou grupos dentro de uma organização, uma comunidade, um meio ou, até mesmo, um acontecimento em especial, como uma mudança política, um conflito (LAVILLE; DIONNE, 1999).

Para a coleta dos dados, foram realizadas entrevistas com três colaboradores (dois gestores gerais e um gestor setorial), em cada uma das três instituições pesquisadas, com o objetivo de obter uma planilha com os principais indicadores adotados pelo programa, a fim de avaliar as mudanças alcançadas por estes e captar informações importantes sobre a construção dos indicadores.

Devido ao grande número de indicadores utilizados pelo programa Pro Hosp foram escolhidos apenas aqueles que versam sobre resultados, ou seja, taxas que demonstram o reflexo da produção hospitalar: Taxa de cesárea, taxa de ocupação, taxa de permanência, taxa de mortalidade e taxa de infecção. 


\section{INDICADORES UTILIZADOS PELO PROGRAMA Pro Hosp: UM REFLEXO DE MUDANÇAS?}

Uma vez pactuados com o programa Pro Hosp, todos os hospitais devem apresentar, periodicamente (o programa é estabelecido por competências anuais), o chamado Relatório Circunstanciado, comprovando a aplicação dos recursos repassados pelo programa. Neste documento também são demonstradas as taxas relativas aos resultados da produção hospitalar e suas mudanças.

Nas Tabelas 1, 2 e 3 observa-se a situação dos hospitais pesquisados, com relação às suas taxas de cesárea, ocupação, permanência, mortalidade e infecção. Os questionamentos e posteriores análises são referentes a mudanças obtidas em relação a todo o tempo de adequação ao programa, ou seja, desde sua adesão até o momento da pesquisa. Com relação às variáveis analisadas: taxas de cesárea, mortalidade, infecção e permanência - estas taxas deveriam ser reduzias, de acordo com o pacto feito entre SES e hospitais participantes do Pro Hosp. Já a taxa de ocupação deveria ser aumentada, pois representa a ociosidade dos leitos disponíveis.

Tabela 1 Situação das taxas referentes ao Hospital X

\begin{tabular}{|c|c|c|c|}
\hline Hospital 1 & Taxa cesárea & Taxa ocupação & Taxa permanência \\
\hline Antes do Pro Hosp & $62 \%$ & --- & \multirow{2}{*}{$\begin{array}{l}\text { Melhora em todas } \\
\text { as clínicas menos } \\
\text { as UTIs. }\end{array}$} \\
\hline \multirow[t]{2}{*}{ Depois do Pro Hosp } & $38 \%$ & Em torno de $80 \%$ & \\
\hline & Taxa mortalidade & Taxa infecção & \\
\hline Antes do Pro Hosp & \multirow{2}{*}{$\begin{array}{l}\text { Houve redução } \\
\text { significativa }\end{array}$} & --- & \\
\hline Depois do Pro Hosp & & Em torno de $2 \%$ a $3 \%$ & \\
\hline
\end{tabular}

Tabela 2 Situação das taxas referentes ao Hospital Y

\begin{tabular}{|c|c|c|c|}
\hline Hospital 2 & Taxa cesárea & Taxa ocupação & Taxa permanência \\
\hline Antes do Pro Hosp & Início, $36,36 \%$ & --- & \multirow{2}{*}{$\begin{array}{l}\text { Exceto clínica médica, } \\
\text { ginecológica, } \\
\text { obstetrícia, } \\
\text { e UTI adulto. }\end{array}$} \\
\hline Depois do Pro Hosp & Hoje, cerca de $80 \%$ & Em torno de $73,29 \%$ & \\
\hline & Taxa mortalidade & Taxa infecção & \\
\hline Antes do Pro Hosp & \multirow{2}{*}{$\begin{array}{c}\text { Cerca de } \\
4,55 \%\end{array}$} & $\begin{array}{c}-- \\
-\end{array}$ & \\
\hline Depois do Pro Hosp & & Em torno de $2,9 \%$ & \\
\hline
\end{tabular}




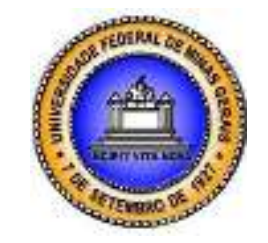

Tabela 3 Situação das taxas referentes ao Hospital Z

\begin{tabular}{l|c|c|c}
\hline \multirow{2}{*}{ Hospital 2 } & Taxa cesárea & Taxa ocupação & Taxa permanência \\
\cline { 2 - 3 } Antes do Pro Hosp & $75 \%$ & Mantida & $\begin{array}{c}\text { Melhora considerável } \\
\text { Clínica médica e } \\
\text { cirúrgica }\end{array}$ \\
\hline Depois do Pro Hosp & $44 \%$ & Em torno de $78,38 \%$ & \\
\cline { 2 - 3 } Antes do Pro Hosp & Taxa mortalidade & Taxa infecção & \\
\cline { 2 - 3 } & Cerca de & --- & \\
\hline Depois do Pro Hosp & $5,9 \%$ & Em torno de $0,75 \%$ & \\
\hline
\end{tabular}

Fonte: Dados da pesquisa

Pode-se perceber que os três hospitais pesquisados alcançaram melhorias expressivas em seus indicadores referentes ao programa Pro Hosp. Há destaque para os Hospitais X e Z, pois estes conseguiram melhores indicadores na maioria das taxas avaliadas.

O Hospital Y não conseguiu manter seu êxito na redução da taxa de cesárea devido a problemas com relação à contratação de uma enfermeira que estaria dedicada à obstetrícia. Além disso, a quebra de um contrato com a prefeitura (que permitia que o hospital atendesse a gestantes provenientes do SUS) acabou por reduzir a base de cálculo para este indicador, uma vez que seu cálculo é realizado mediante a relação percentual entre o número de partos cirúrgicos realizados em determinado período e o número total de partos realizados (normais e cirúrgicos) do mesmo período.

A taxa de ocupação calculada por meio da relação entre o número de pacientes-dia em determinado mês e o número de leitos-dia do mesmo período, foi mantida entre $73,29 \%$, no Hospital Y; 78,38\%, no Hospital Z e cerca de 80\%, no Hospital X. Esses valores corroboram a perspectiva defendida por La Forgia e Coutollenc (2009) de que a taxa desejável de ocupação de leitos deve estar entre $75 \%$ e $85 \%$. Percebe-se que há uma conformidade entre as informações obtidas nos três hospitais, o que, provavelmente, é reflexo dos recursos financeiros repassados pelo programa e que são utilizados na renovação da estrutura, como a construção de novos leitos.

A taxa de permanência também foi melhorada em todos os hospitais, ficando as UTIs de fora destas mudanças, devido ao fato de que esta área do hospital recebe pacientes em estados mais críticos e que, portanto, acabam ficando mais tempo no leito. Esta taxa, também chamada tempo médio de permanência, é obtida por meio da relação entre o número de pacientes-dia em determinado mês e o número de saídas hospitalares no mesmo período. Deste modo, a melhoria nesta taxa reflete controles adotados para que o paciente, quando possível, não fique dias em excesso no hospital, seja por qualquer razão. As causas que são de responsabilidade da instituição são tratadas, estando intimamente ligadas à taxa de infecção, à 

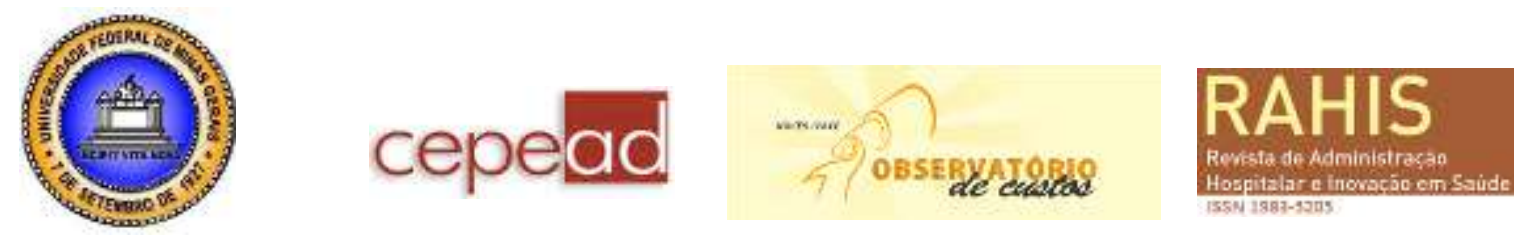

segurança no leito e ao controle de diárias pagas por convênios particulares e SUS (Sistema Único de Saúde).

Com relação à taxa de mortalidade, apenas os hospitais $\mathrm{Y}$ e $\mathrm{Z}$ declararam seus indicadores, ficando com 4,55\% e 5,9\%, respectivamente. O cálculo desta taxa é estabelecido pela relação percentual entre o número de óbitos ocorridos no hospital em determinado mês e o número de saídas do mesmo período. Já a taxa de infecção, uma das mais importantes, foi mantida entre $0,75 \%$ (Hospital Z) até 3\% (Hospital X). O cálculo desta taxa é realizado por meio da relação percentual entre os números de episódios de infecção hospitalar em determinado mês e o total de saídas do mesmo período.

A falta de informações anteriores sobre os diversos índices demonstram que o programa Pro Hosp, além de incentivar melhorias, foi capaz de ser o indutor na conformação de um sistema de informações preponderantes para a consecução da atividade hospitalar. É preciso lembrar que todas as informações trazidas pelos indicadores são baseadas em um complexo sistema de coleta e observação, o chamado censo hospitalar. Trata-se de um documento que contém várias estatísticas consideradas vitais para a prestação de serviços de saúde, como número de leitos vagos e ocupados e contagem e registro do número de internações, altas, óbitos, transferências internas e externas ocorridas em um espaço de tempo de 24 horas.

Para que este sistema funcione da maneira mais fidedigna possível, é preciso haver a colaboração entre os funcionários envolvidos no processo de atendimento ao paciente.

\section{CONSIDERAÇÕES FINAIS}

As PPP's são um tipo de relacionamento interorganizacional, baseado na contratualização entre Estado e entidades privadas, o que pode ser observado na aplicação da NGP ao estado de Minas Gerais, especificamente às ações estratégicas direcionadas à saúde. O programa Pro Hosp, dedicado à reestruturação dos hospitais filantrópicos de Minas Gerais, insere-se nesta perspectiva, pois tem como meio de ação o estabelecimento de contratos de metas e objetivos entre hospitais e a SES. Estes contratos contemplam e incentivam melhorias em diversas áreas e estas são mensuradas por meio de indicadores de desempenho.

Como incentivo às mudanças, os hospitais recebem recursos financeiros repassados mediante o cumprimento das metas estabelecidas. De modo geral, o objetivo do programa é afetar positivamente a prestação de serviços de saúde por parte do SUS, por meio de melhorias direcionadas à estrutura do hospital, aos seus processos alocativos, à própria gestão da instituição e ao reconhecimento da importância de resultados mais eficientes.

Para sua consecução, o programa utiliza indicadores de desempenho hospitalar, a fim de avaliar e acompanhar as metas e as mudanças propostas pelo acordo. Estes indicadores podem ser divididos em cinco diferentes áreas, abrangendo vários aspectos da dinâmica organizacional.

A área que se refere aos resultados da produção hospitalar abrange taxas como as de mortalidade, permanência, infecção, cesárea e ocupação. Uma análise sobre as informações veiculadas por estas taxas, obtidas em três instituições hospitalares participantes do Pro Hosp, revela, de maneira geral, melhorias em índices que puderam ser comparadas. Além disso, revela também que não havia um rígido sistema de informações baseado no censo hospitalar anteriormente ao programa. Em alguns indicadores, as instituições não souberam indicar suas 

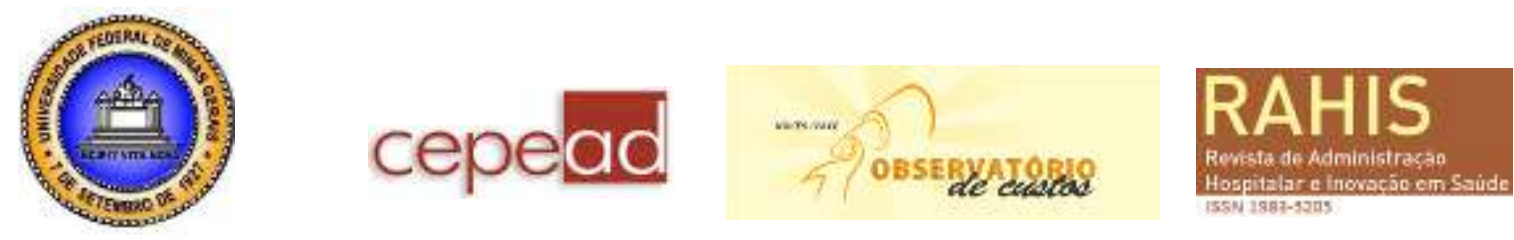

taxas antes da pactuação com o programa, o que corrobora a ideia de que o Pro Hosp, além de melhorias nestes indicadores, também acabou agindo na formação de um sistema de informações importantes para as decisões organizacionais.

\section{REFERÊNCIAS}

AKINTOYE, A.; BECK, M.; HARDCASTLE, C. Public private partnerships: managing risks and opportunities. Oxford: Blackwell Science, 2003. 448 p.

ALCOFORADO, F. C. G. Contratualização e eficiência no setor público: as organizações sociais. In: SEMINÁRIO ORGANIZAÇÃ̃O SOCIAL (OS) E ORGANIZAÇÃO CIVIL DE INTERESSE PÚBLICO (OSCIP) DE CULTURA, 2., 2007, São Paulo. Anais... São Paulo: Secretaria de Economia e Planejamento, 2007. p. 109-135.

ALONSO-CONDE, A. B.; BROWN, C.; ROJO-SUAREZ, J. Public private partnerships: incentives, risk transfer and real options. Review of Financial Economics, Califórnia, v. 16, p. 335-349, Apr. 2007.

ANDERSEN, J. F. Os indicadores sociais como instrumento de promoção do desenvolvimento intra-municipal. 2004. 62 p. Dissertação (Mestrado em Engenharia de Produção)-Universidade Federal de Santa Catarina, Florianópolis, 2004.

BULT-SPIERING, M.; DEWULF, G. Strategic issues in public-private partnerships: an international perspective. 2. ed. London: Wiley Blackwell, 2007. 216 p.

EVENHUIS, E.; VICKERMAN, R. Transport pricing and public-private partnerships in theory: issues and suggestions. Research in Transportation Economics, Oregon, v. 30, n. 1, p. 6-14, Jan. 2010.

FREDEBEUL-KREIN, M.; KNOBEN, W. Long term risk sharing contracts as an approach to establish public-private partnerships for investment into next generation access networks. Telecommunications Policy, Oxford, v. 34, n. 9, p. 528-539, Oct. 2010.

GALVÃO, M. C. C. P. (Coord.) Novas formas de gestão dos serviços públicos: a relação público-privada. São Paulo: FUNDAP, 1997. 68 p.

GHALAYINI, A. M.; NOBLE, J. S. The changing basis of performance measurement. International Journal of Operations \& Production Management, West Yorkshire, v. 16, n. 8, p. 63-80, 1996.

INSTITUTO BRASILEIRO DE GEOGRAFIA E ESTATÍSTICA. Estatísticas da saúde: Assistência Médico-Sanitária (AMS) 2009. Rio de Janeiro, 2010. 167 p. 

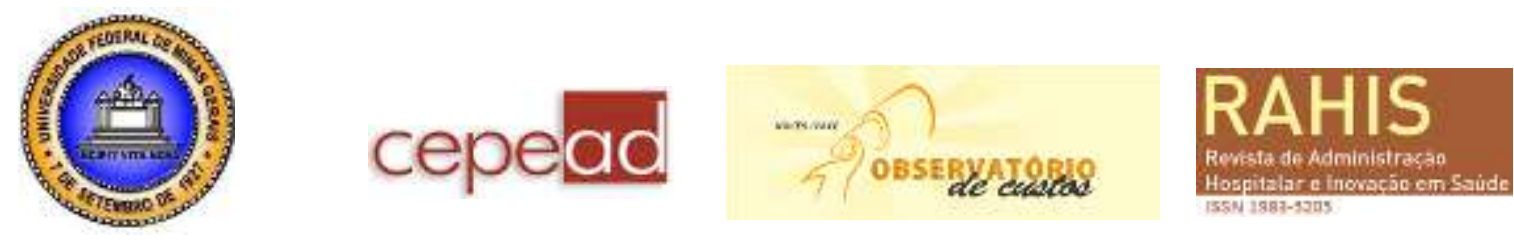

JONG, M. et al. Introducing public-private partnerships for metropolitan subways in China: what is the evidence? Journal of Transport Geography, Oxon, v. 18, n. 2, p. 301-313, Mar. 2010.

KE, Y. et al. Preferred risk allocation in China's public-private partnership (PPP) projects. International Journal of Project Management, Guildford, v. 28, n. 5, p. 482-492, July 2010.

KHAN, S.; MUSHTAG, S. Regional partnerships to assist public-private investments in irrigation systems. Agricultural Water Management, Amsterdam, v. 96, n. 5, p. 839-84, May 2009.

KLIJN, E. H.; TEISMAN, G. R. Institutional and strategic barriers to public-private partnership: an analysis of dutch cases. Public Money and Management, Oxfordshire, v. 23, n. 3, p. 137-146, July 2003.

LA FORGIA, G. M.; COUTTOLENC, B. F. Desempenho hospitalar no Brasil: em busca da excelência. São Paulo: Singular, 2009. 446 p.

LANE, J. -E. New public management. London: Routledge, 2000. 256 p.

LAVILLE, C.; DIONNE, J. As estratégias de verificação. In: A construção do saber. Belo Horizonte: UFMG, 1999. p. 131-163.

LIMA, H. O. et al. Fortalecimento e qualificação da rede hospitalar - Pro Hosp. In: MARQUES, A. J. de S. et al. (Org.). O choque de gestão na saúde em Minas Gerais. Belo Horizonte: Secretaria do Estado de Saúde de Minas Gerais, 2009. p. 131-157.

MARTINS, A. C.; MARQUES, R. C.; CRUZ, C. O. Public-private partnerships for wind power generation: the portuguese case. Energy Policy, Oxon, v. 39, n. 1, p. 94-104, Jan. 2011.

MAYSTON, D. J. The private finance initiative in the national health service: an unhealthy development in new public management? Financial Accountability \& Management, Oxford, v. 15, n. 3, p. 249-274, Aug./Nov. 1999.

MENDES, E. V. O Pro Hosp. In: MARTINS, M. M.; COUTINHO, S.; SANTOS, A. (Org.). Caderno de especialização em gestão hospitalar para o Pro Hosp. Belo Horizonte: ESPMG, 2007. p. 9-12.

MENDES, E. V.; SILVA, M. V. C. P. da. Pacto de gestão: da municipalização autárquica à regionalização cooperativa. Belo Horizonte: Secretaria de Estado de Saúde de Minas Gerais, 2004. $80 \mathrm{p}$. 

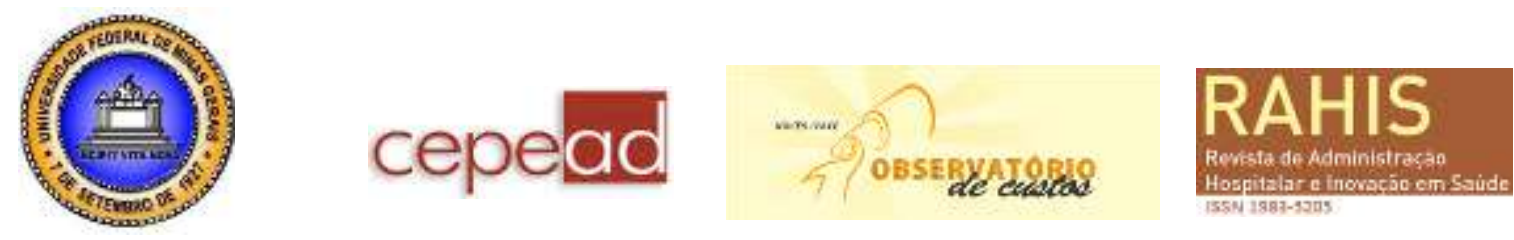

MINAS GERAIS. Secretaria de Estado de Saúde. Manual do Programa de Fortalecimento e Melhoria da Qualidade dos Hospitais do SUS/MG (Pro Hosp). Belo Horizonte: Secretaria do Estado de Minas Gerais/Fundação João Pinheiro, 2007. 100 p.

MOLINA, C. G. Modelo de formación de políticas y programas sociales. Washington, 2002. Notas de clase. Curso Diseño Y Gerencia de Políticas y Programas Sociales. INDES Instituto Interamericano para el Desarrollo Social.

MOREIRA NETO, D.F.M. Parcerias Público-Privadas e Responsabilidade Fiscal: uma conciliação possível (Prefácio). Rio de Janeiro: Lumen Juris Editora, 2005.

MU, R.; JONG, M.; KOPPENJAN, J. The rise and fall of public-private partnerships in China: a path-dependent approach. Journal of Transport Geography, Oxon, v. 19, n. 4, p. 794-806, Dec. 2011.

NARROD, C. et al. Public-private partnerships and collective action in high value fruit and vegetable supply chains. Food Policy, Oxon, v. 34, n. 1, p. 8-15, 2009.

NEELY, A.; BOURNE, M. Why measurement initiatives fail. Measuring Business Excellence, Bradford, v. 4, n. 4, p. 3-7, Dec. 2000.

PASIN, J. A. B.; BORGES, L. F. X. A nova definição de parceria público-privada e sua aplicabilidade na gestão de infraestrutura pública. Revista do BNDES, Rio de Janeiro, v. 10, n. 20, p. 173-196, dez. 2003.

PECI, A.; SOBRAL, F. Parcerias público-privadas: análise comparativa da experiência inglesa e brasileira. In: ENCONTRO DA ASSOCIAÇÃO NACIONAL DE PÓS GRADUAÇÃO E PESQUISA EM ADMINISTRAÇÃO, 30., 2006, Salvador, BA. Anais... Salvador: ENANPAD, 2006. p. 1-17.

POULTON, C.; MACARTNEY, J. Can public-private partnerships leverage private investment in agricultural value chains in Africa? A preliminary review. World Development, Oxford, v. 40, n. 1, p. 96-109, June 2011.

QUAH, V.; LIM, C. P.; BROOK, C. Public-private partnerships for education reform. In: PETERSON, P.; BAKER, E.; MCGRAW, B. International Encyclopedia of Education. 30. ed. Oxford: Elsiever, 2010, p. 73-80.

RUSSELL, S.; BENNETT, S.; MILLS, A. Reforming the health sector: towards a health new public management. Journal of International Development, Chichester, v. 11, n. 5, p. 765775, Aug./July 1999.

SOARES, R. P.; CAMPOS NETO, C. A. da S. Parcerias público-privadas do Plano Plurianual: proposta de um conceito. Brasília: IPEA, 2002. 51 p. (Texto para discussão, n. 929). 
SUNDFELD, C. A. O arcabouço normativo das parcerias público-privadas no Brasil. Revista do TCU, Brasília, n. 104, p. 53-61, abr./jun. 2005.

TAKASHIMA, R.; YAGI, K.; TAKAMORI, H. Government guarantees and risk sharing in public-private partnerships. Review of Financial Economics, New Orleans, v. 19, n. 2, p. 78-83, Apr. 2010.

TANG, L.; SHEN, K.; CHENG, E. W. L. A review of studies on public-private partnership projects in the construction industry. International Journal of Project Management, Guildford, v. 28, n. 7, p. 683-694, 2010.

VERGARA, S. Projetos e relatórios de pesquisa em administração. São Paulo: Atlas, 2000. 102 p. 